\title{
Primeiro relato de Platynosomum spp. em um felino doméstico no estado do Paraná, Brasil
}

\author{
[First report of Platynosomum spp. in a domestic cat in the state of Paraná, Brazil]
}

\section{"Relato de Caso/Case Report"}

\author{
Yohana Gonçalves Vieira ${ }^{\mathbf{1}^{*}}$, Gabriela Maria Benedetti Vasques ${ }^{2}$, Thalita Regina Petrillo ${ }^{2}$, Juliana \\ Evangelista Bezerril ${ }^{\mathbf{3}}$, Andressa de Cassia Rais ${ }^{\mathbf{1}}$, Rafaela Bonfim Melo ${ }^{\mathbf{1}}$, \\ Paulo Fernandes Marcusso ${ }^{4}$
}

\author{
${ }^{1}$ Médica Veterinária Autônoma, Maringá-PR, Brasil. \\ ${ }^{2}$ Departamento de Medicina Veterinária, Centro Universitário Ingá, Maringá-PR, Brasil. \\ ${ }^{3}$ Departamento de Medicina Veterinária, Centro Universitário de Mineiros-GO, Brasil. \\ ${ }^{4}$ Departamento de Medicina Veterinária, Instituto de Ciências Agrárias, Universidade Federal dos Vales do \\ Jequitinhonha e Mucuri, Unaí-MG, Brasil. \\ *Autor para correspondência/Corresponding author: E-mail: yohana-vieira@ hotmail.com
}

\section{Resumo}

Existem poucos trabalhos descritos sobre infecção por Platynosomum spp. em felinos domésticos, e seus sinais clínicos são muitas vezes inespecíficos. Entretanto, essa patologia é de grande importância na clínica felina e deve estar na lista de diagnósticos diferenciais de patologias do aparelho hepatobiliar. As informações retratadas relatam um caso clínico de platinosomíase por meio de características clínicas, hematológicas, ultrassonográficas, necroscópicas e histopatológicas em um felino doméstico, macho, quatro anos de idade, com acesso à rua, com sinais clínicos de hiporexia, prostração, tentativas de vômitos e mudanças na característica das fezes, diagnosticado com platinosomíase, sendo este, o primeiro registro de platinosomíase no estado do Paraná-Brasil.

Palavras-chave: alteração hepatobiliar; Felis catus; platinosomíase; platinosomose; trematódeo.

\begin{abstract}
There are few studies on Platynosomum spp. infection in domestic cats, and its clinical signs are often nonspecific. However, this pathology is of great importance in the feline clinic and should be on the list of differential diagnoses for pathologies of the hepatobiliary system. The information described report a clinical case of platynosomiasis via clinical, hematological, ultrasound, necroscopic, and histopathological characteristics in a 4-year-old male cat with access to the street, with clinical signs of hypoxia, prostration, dry heaving, and changes in feces characteristics, diagnosed with platynosomiasis, being the first case reported of platynosomiasis in the state of Parana-Brazil.
\end{abstract}

Keywords: hepatobiliary alteration; Felis catus; platynosomiasis; platynosomosis; trematode.

\section{Introdução \\ Platynosomum spp. (Trematoda) é encontrado em áreas tropicais e subtropicais no mundo, que pode parasitar o fígado, ductos biliares e vesícula biliar e em menor frequência outros tecidos, como o intestino delgado, ductos pancreáticos e pulmão de gatos domésticos (Felis}

catus) (Pimentel et al., 2005; Basu e Charles, 2014).

Este trematódeo é considerado o parasito hepático mais importante dos felinos domésticos (Ferreira e Almeida, 2003). Em áreas endêmicas pode acometer de 15 a $85 \%$ dos gatos (Norsworthy, 2003). 
No Brasil existem relatos de felinos acometidos nos estados de São Paulo (Xavier et al., 2007), Minas Gerais (Vieira et al., 2009), Rio Grande do Sul (Michaelsen et al., 2012), Bahia (Rocha et al., 2014; Jesus et al., 2015), Ceará (Sousa Filho et al., 2015), Pernambuco (Ramos et al., 2016), Rio de Janeiro (Carvalho et al., 2017), Maranhão (Silva et al., 2017), Espírito Santo (Campos et al., 2018), e Tocantins (Sobral et al., 2019).

Há relatos de $P$. fastosum, $P$. illiciens e $P$. concinnum como parasitos das vias biliares de gatos domésticos e, portanto, acredita-se que as três espécies supracitadas são consideradas sinonímias (Salomão et al., 2005; Soldan e Marques, 2011; Basu e Charles, 2014; Corrêa et al., 2016).

O ciclo de vida do parasito necessita de três hospedeiros intermediários (moluscos, crustáceos e vertebrados inferiores, como sapos e lagartixas) antes de acometer o hospedeiro definitivo (felinos domésticos e selvagens) (Shell et al., 2015).

A maioria dos animais são hospedeiros assintomáticos, mas quando ocorrem sinais clínicos eles estão relacionados com o grau do parasitismo, tempo de infecção e relação individual ao parasito (Pimentel et al., 2005). Os principais sinais são êmese, efusão abdominal, hepatomegalia, anorexia, letargia, anemia, icterícia e perda de peso. Em casos severos pode ocorrer obstrução do fluxo biliar, os animais apresentam diarreia com muco e mudança da característica das fezes (Soldan e Marques, 2011, Montserin et al., 2013).

Devido aos sinais clínicos inespecíficos para esta patologia, as técnicas de diagnóstico em gatos com platinosomíase são essencialmente importantes na epidemiologia desse parasitismo (Michaelsen et al., 2012). O diagnóstico definitivo é feito pela detecção de ovos operculados nas fezes (Michaelsen et al., 2012), associado a exames complementares como ultrassonografia abdominal, hemograma e perfil bioquímico, uma vez que o diagnóstico coproparasitológico não confere total eficácia na confirmação da doença (Michaelsen et al., 2012).

No exame ultrassonográfico em casos de platinosomíase, é possível notar hepatomegalia, alterações de ecotextura hepática, também se observa espessamento da parede da vesícula biliar com lama ou barro biliar. Quando a carga parasitária é alta, é notado dilatação dos ductos biliares, distensão da vesícula biliar e até fibrose periductal (Mamprim e Sartor, 2016).
São achados comuns de necropsias em gatos acometidos, alterações inflamatórias do ducto biliar comum e ducto pancreático. A obstrução pode ser causada por coincidência ou consequência da colangiohepatite (Soldan e Marques, 2011; Braga et al., 2016). A possível causa da icterícia em casos de platinosomíase é a associação do parasitismo com a fibrose hepática, ocasionando o impedimento do fluxo da bile para o duodeno (Sampaio et al., 2006).

O tratamento indicado para essa parasitose é o anti-helmíntico praziquantel (Michaelsen et al., 2012), que pode ser administrado por via subcutânea ou via oral, indicado na dose de 20 $\mathrm{mg} / \mathrm{kg}$ pela via subcutânea, uma vez ao dia, durante três a cinco dias. Em outro protocolo, o praziquantel é indicado na dose de $10 \mathrm{mg} / \mathrm{kg}$ pela via oral, durante três dias. A terapia deve ser repetida no intervalo de 12 semanas (Ferreira e Almeida, 2003).

O objetivo deste trabalho foi descrever as características clínicas, sonográficas e histopatológicas de um felino com platinosomíase atendido na cidade de Maringá, estado do Paraná, Brasil.

\section{Descrição do Caso}

Foi atendido no dia 20 de maio de 2015, no setor de clínica médica em um Hospital Veterinário na cidade de Maringá, estado do Paraná, um felino, macho, sem raça definida, com quatro anos de idade, pesando $3,7 \mathrm{Kg}$. A queixa principal consistia em hiporexia, prostração, tentativas de vômitos e mudanças na característica das fezes. O tutor também relatou vacinação atrasada, nenhuma everminação, acesso à rua e hábito de caçar lagartixas.

No exame físico foram observadas mucosas oral e conjuntival ictéricas, temperatura corpórea de $38^{\circ} \mathrm{C}$, frequência cardíaca de $160 \mathrm{bpm}$, frequência respiratória de $36 \mathrm{mpm}$ e linfonodos submandibulares e poplíteos dentro da normalidade para espécie, paciente apresentandose apático, mas com propriocepção.

$\mathrm{O}$ animal foi internado para realização de exames complementares, como ultrassonografia abdominal e análise hematológica. $\mathrm{Na}$ análise hematológica, o eritrograma não apresentou alteração, o leucograma apresentou leucocitose por neutrofilia $(12.933 \mu \mathrm{L})$, eosinofilia $(3.776 \mu \mathrm{L})$, e monocitose $(2.018 \mu \mathrm{L})$. O trombograma também não apresentou alterações. As avaliações bioquímicas revelaram aumento no nível sérico da 
enzima fosfatase alcalina ( $400 \mathrm{U} / \mathrm{L})$, do composto nitrogenado uréia $(394,7 \mathrm{mg} / \mathrm{dL})$, da bilirrubina total $(8,2 \mathrm{mg} / \mathrm{dL})$ e da bilirrubina direta $(6,1$ $\mathrm{mg} / \mathrm{dL})$. As concentrações séricas da enzima alanina aminotransferase $(59 \mathrm{U} / \mathrm{L})$, da creatinina $(0,8 \mathrm{mg} / \mathrm{dL})$ e das proteínas totais plasmáticas $(6,8$ $\mathrm{g} / \mathrm{dL}$ ) normais, entretanto a concentração de albumina $(2,0 \mathrm{~g} / \mathrm{dL})$ estava abaixo dos valores de referência, enquanto a de globulina estava acima $(4,8 \mathrm{~g} / \mathrm{dL})$.

Na ultrassonografia foi possível observar o fígado com dimensões aumentadas, contornos regulares e parênquima homogêneo hipoecogênico, compatível com hepatopatia inflamatória. A vesícula biliar acentuadamente repleta, parede espessada e conteúdo anecogênico com sedimento ecogênico, indícios de lama biliar. Observou-se também acentuada dilatação de ductos hepáticos e ducto cístico, sinais de processo inflamatório e/ou obstrutivo. De acordo com os sinais supracitados considerou-se colangitecolangiohepatite. $\mathrm{O}$ estômago apresentou espessamento discreto a moderado de parede.

Com os resultados dos exames laboratoriais hematológicos, associados aos sinais clínicos, histórico de acesso à rua e hábito de caçar lagartixas, suspeitou-se de platinosomíase e iniciou-se o tratamento contra parasitose, para tanto foi prescrito a terapia com o anti-helmíntico praziquantel na dose de $10 \mathrm{mg} / \mathrm{kg}$ a cada 24 horas durante 3 dias.

Após 2 dias de tratamento, no dia 22 de maio de 2015, o animal não apresentou melhora e foi à óbito, e no mesmo dia foi realizada a necropsia. No exame necroscópico externo o animal apresentava boa condição corporal, com ampla área de tricotomia na região ventral, intensa icterícia na pele e mucosas oral e conjuntivais (Figura 1A).

Após incisão da pele sobre a linha média observou-se icterícia no tecido adiposo subcutâneo. $\mathrm{Na}$ abertura da cavidade abdominal foi possível observar intensa icterícia na gordura mesentérica e no tecido adiposo abdominal. O fígado apresentava aumento de tamanho moderado, coloração amarelada, vesícula biliar repleta, evidenciação do padrão lobular e com pequenos nódulos $(0,1$ a 0,3 $\mathrm{cm})$ de consistência firme disseminados no lobo medial esquerdo e nas bordas dos lobos lateral esquerdo e medial direito (Figura 1B).

Antes da separação do fígado do intestino delgado foi realizada a manobra de Virchow, ou seja, realizou-se abertura da porção proximal do duodeno na região antimesentérica até o piloro e pressionou-se levemente a vesícula biliar até o extravasamento ou não de bile no intestino delgado, resultando no não extravasamento da bile (Figura 1C).

Após separação do fígado foi possível observar intensa dilatação dos ductos biliares na face caudal do órgão (Figura 1D). Ao corte, o fígado drenou líquido amarelo-esverdeado discretamente viscoso, observou-se dilatação da espessura dos ductos biliares no parênquima do órgão. A vesícula biliar foi aberta e apresentou conteúdo extremamente viscoso e de coloração verde escura (Figura 1C).

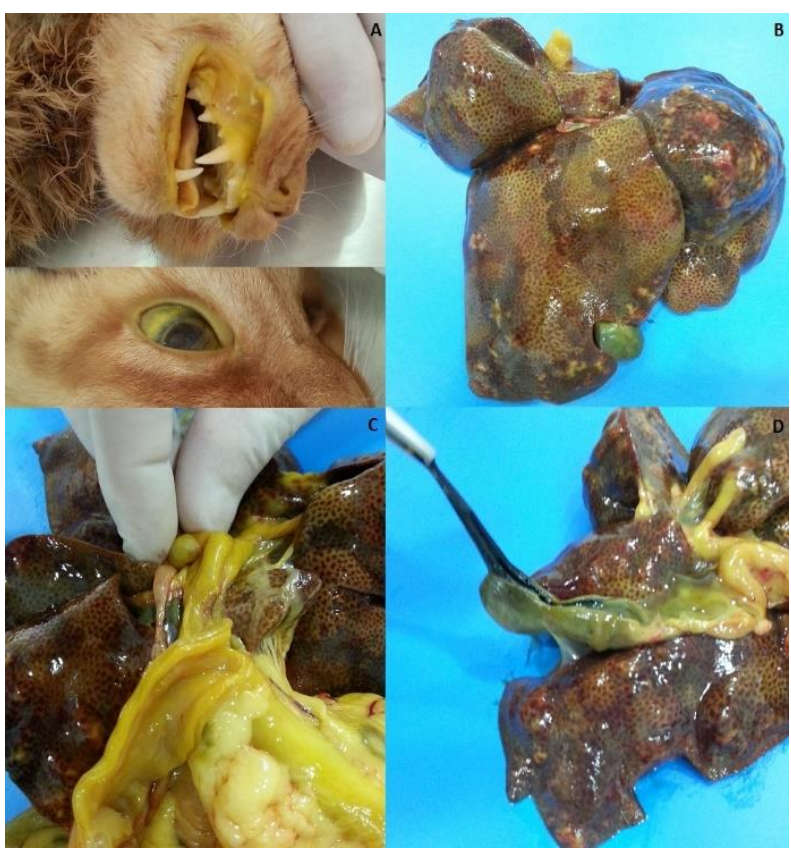

Figura 1. Necropsia de felino. A) Intensa icterícia nas mucosas oral e conjuntivais. B) Hepatomegalia moderada, vesícula biliar repleta e evidenciação do padrão lobular com pequenos nódulos $(0,1$ a $0,3 \quad \mathrm{~cm})$ de consistência firme disseminados no lobo medial esquerdo e nas bordas dos lobos lateral esquerdo e medial direito. C) Manobra de Virchow negativa. D) Dilatação dos ductos biliares e conteúdo biliar na extremidade da pinça com extrema viscosidade e de coloração verde escura

Durante a necropsia foi realizada a coleta de fragmentos do fígado, pâncreas, intestino delgado e grosso, estômago, rins, coração, pulmão e encéfalo para processamento de rotina e análise histopatológica.

A análise histológica hepática revelou discreto exsudato misto (neutrófilos, eosinófilos e macrófagos) e intensa hiperplasia dos ductos biliares, acompanhado de acentuada fibrose do 
tecido conectivo ao redor dos ductos biliares, com infiltrado inflamatório mononuclear periductal e visualização de secções do parasito na luz do ducto (Figura 2). Além de discreta congestão hepática, infiltrado mononuclear perivascular e hepatócitos com discreta degeneração hidrópica periportal.

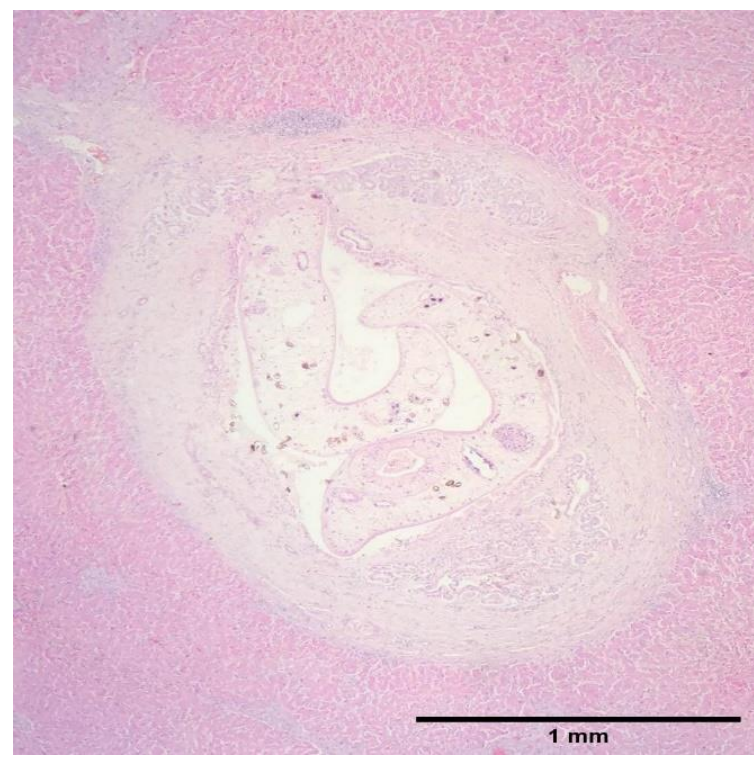

Figura 2. Histopatologia hepática de felino evidenciando secções do parasito Platynosomum spp., na luz do ducto biliar (H\&E e $1 \mathrm{~mm}$ barra). Objetiva de $10 x$.

\section{Discussão}

A faixa etária, sexo e estilo de vida do felino se relacionam com a possibilidade da infecção, informação que é descrita por Soldan e Marques (2011) e Köster et al. (2016), que indicaram que os felinos acima de dois anos de idade, que possuem vida livre ou são semiconfinados (acesso à rua), e com hábito de caçar lagartixas apresentam maior incidência de platinosomíase. Entretanto, Soldan e Marques, (2011) e Köster et al., (2016), referiram fêmeas com maior ocorrência de infecção quando comparadas aos machos.

O hemograma apresentava uma leucocitose com discreta neutrofilia, acentuada eosinofilia e monocitose. A leucocitose pode ser considerada inflamatória, pois mesmo a neutrofilia não apresentando desvio a esquerda, ela está acompanhada de monocitose, característica de processo inflamatório que no caso seu deu pela presença do parasito nos ductos biliares (Weiser e Thrall, 2007). O parasitismo também gerou uma resposta celular com aumento de eosinófilos pela própria característica efetora dessas células contra parasitos (Stockham e Scott, 2011).
No exame bioquímico sérico foi possível observar um aumento cinco vezes maior da enzima fosfatase alcalina ( $400 \mathrm{U} / \mathrm{L}$ ) em relação aos valores de referência da espécie. Apesar da fosfatase alcalina não ser uma enzima de alta especificidade hepática encontrada também nos tecidos ósseo, renal, intestinal e nas hemácias, não há em nosso caso alterações nesses locais que justifiquem tal aumento, apenas as lesões hepáticas diagnosticadas pelos exames ultrassonográfico e necroscópico que poderiam justificar um aumento dessa magnitude (Stockham e Scott, 2011).

A concentração sérica de ureia (394,7 $\mathrm{mg} / \mathrm{dL}$ ) também aumentou em relação aos valores de referência. Esse aumento pode estar relacionado ao acumulo de ureia na bile, pois as junções oclusivas dos hepatócitos são relativamente permeáveis e diversas substâncias podem passar por difusão (passivamente) do plasma sanguíneo para bile, sendo uma delas a ureia, uma vez que a função excretora renal estava preservada, pois a concentração sérica de creatinina $(0,8 \mathrm{mg} / \mathrm{dL})$ estava dentro dos valores de referência. Outra hipótese para o aumento da ureia está relacionada ao comprometimento da alimentação do animal associado à mudança do trânsito gastrointestinal $\mathrm{e}$ características das fezes, como citada na anamnese pelo proprietário. Essas alterações podem induzir à alteração na microbiota normal do intestino, levando a multiplicação de bactérias produtoras de amônia que foi convertida pelos hepatócitos em ureia, se acumulando no sangue, haja vista que a função dos hepatócitos estava preservada já que a concentração sérica de alanina aminotransferase (59 U/L) estava dentro dos valores de referência da espécie (Stockham e Scott, 2011).

Os achados do exame ultrassonográfico foram os semelhantes aos citados por outros autores que diagnosticaram gatos domésticos com platinosomíase, como: fígado com dimensões aumentadas, contornos regulares e parênquima homogêneo hipoecogênico, sinais de hepatopatia inflamatória (Xavier et al., 2007), vesícula biliar acentuadamente repleta, parede espessada e conteúdo anecogênico com sedimento ecogênico, indícios de formação de lama biliar (Haney et al., 2006). Observou-se também moderada a acentuada dilatação de ductos hepáticos e ducto cístico, sinais de processo inflamatório e/ou obstrutivo, já descritos por Sampaio et al. (2006).

Segundo Salomão et al. (2005) as alterações hepáticas observadas no exame ultrassonográfico de gatos positivos para Platynosomum sp., quando 
avaliadas isoladamente, não servem como diagnóstico para a infecção, mas a associação de achados de lesões da vesícula biliar e das vias biliares e anamnese pode ser indicativos de platinosomíase, semelhante ao relato, onde o histórico de caçar lagartixas aliado ao exame de imagem e laboratorial foi determinante para o diagnóstico presuntivo.

As alterações macroscópicas da necropsia indicam dilatação dos ductos biliares com acúmulo de bile, aumento da espessura dos ductos biliares, icterícia na pele, mucosas, tecido adiposo subcutâneo, gordura mesentérica e tecido adiposo abdominal condizentes com o encontrado por Ramos et al. (2017). Também foi possível observar a vesícula biliar repleta de bile também relatado por Norsworthy (2003). Essas alterações citadas correspondem as mesmas já descritas por Salomão et al. (2005).

A manobra de Virchow negativa corresponde a obstrução e congestão hepática, um forte indicativo de platinosomíase. Neste caso, pode ter ocorrido pelos próprios parasitos no lúmen do ducto, associado ao processo inflamatório. Tal obstrução culminou no acúmulo de bile que levou a icterícia. O fígado ictérico e com seu tamanho aumentado com evidenciação do padrão lobular e com pequenos nódulos de consistência firme presentes corresponde ao mesmo encontrado por Sousa Filho et al. (2015).

O tratamento foi realizado com a prescrição do anti-helmíntico praziquantel na dose de $10 \mathrm{mg} / \mathrm{kg}$ a cada 24 horas, durante três dias, conforme descreveram Ferreira e Almeida (2003), porém, o felino do relato apresentava severo acometimento hepático, sugestivos pelos exames complementares e confirmado pelo exame necroscópico externo, ressaltando o que foi descrito por Soldan e Marques (2011), que o sucesso do tratamento depende do grau e extensão dos danos causados pelo parasito ao fígado, vesícula biliar e ductos biliares, ocorridos até o momento do diagnóstico, sugerindo que quanto mais cedo o diagnóstico e menor o acometimento hepatobiliar, maiores são as chances da resolução do quadro clínico e menores as chances de óbito.

O exame histopatológico foi definitivo para confirmação da suspeita clínica, na qual foi possível observar o parasito na luz do ducto biliar, além das alterações causadas por ele, como a intensa hiperplasia dos ductos e acentuada fibrose do tecido conectivo ao redor dos ductos biliares com infiltrado inflamatório mononuclear periductal (Sousa Filho et al., 2015; Ramos et al., 2017).

O exame parasitológico das fezes para detecção de ovos não foi utilizado pela baixa sensibilidade, já que nos casos os quais há obstrução dos ductos biliares haverá pouca ovoposição e de forma intermitente (Köster et al. 2016).

\section{Conclusão}

Neste primeiro relato de platinosomíase no estado do Paraná, o exame histopatológico foi essencial para a confirmação da suspeita clínica, em associação com a anamnese detalhada sobre histórico de caça de lagartixas, e exames complementares como a ultrassonografia, bioquímica sérica e os sinais clínicos compatíveis com essa afecção. Ressalta-se, assim, a importância dessa patologia na rotina clínica felina e como deve estar na lista de diagnósticos diferenciais em alterações do sistema hepatobiliar.

\section{Conflito de Interesse}

Os autores declaram não existir conflito de interesse.

\section{Referências}

Basu, A.K.; Charles, R.A. A review of the cat liver fluke Platynosomum fastosum Kossack, 1910 (Trematoda: Dicrocoeliidae). Veterinary Parasitology, 200(1): 1-7, 2014.

Braga, R.R.; Teixeira, A.C.; Oliveira, J.A.; Cavalcanti, L.P. Prevalence of Platynosomum fastosum infection in free roaming cats in northeastern Brazil: fluke burden and grading of lesions. Veterinary Parasitology, 227: 20-25, 2016.

Carvalho, T.K.; Batista, L.S.O.; Sampaio, L.A.L.; Aragão, A.P. Diagnóstico anatomohistopatológico de platinosomose em felino: Relato de caso. Acta Biomedica Brasiliensia, 8(2): 140-146, 2017.

Campos, N.C.; Siqueira, D.F.; Perin, L.R.; Oliveira, L.C.; Campos, D.R.; Martins, I.V.F. Infecção natural por Platynosomum fastosum em felino doméstico no município de Alegre, Espírito Santo e sucesso no tratamento com praziquantel. Medicina Veterinária (UFRPE), 12(1): 17-21, 2018.

Corrêa, P.; Bueno, C.; Vieira, F.M.; Muniz-Pereira, L.C. Helminth parasites of Galictis cuja (Carnivora, Mustelidae), from localities in the Atlantic forest of Brazil. Revista Brasileira de 
Parasitologia Veterinária, 25(4): 407-413, 2016.

Ferreira A.M.R.; Almeida, E.C.P. Platinosomose. In: Souza, H.J.M. Coletâneas em medicina e cirurgia felina. $1^{\text {a }}$ ed. Rio de Janeiro: L.F. Livros de Veterinária, 2003. 2980p.

Haney, D.R.; Christiansen, J.S.; Toll, J. Severe cholestatic liver disease secondary to liver fluke (Platynosomum concinnum) infection in three cats. Journal of the American Animal Hospital Association, 42(3): 234-237, 2006.

Jesus, M.F.P.; Brito, J.A.; Silva, V.C.; Pedroso, P.M.O.; Pimentel, L.A.; Macedo, J.T.S.A.; Santin, F.; Silva, S.M.; Neto, A.F.S.; Ribeiro, R.R. Natural infection by Platynosomum illiciens in a stray cat in Cruz das Almas, Recôncavo da Bahia, Brazil. Brazilian Journal of Veterinary Pathology, 8(1): 25-28, 2015.

Köster, L.; Shell, L.; Illanes, O.; Lathroum, C.; Neuville, K.; Ketzis, J. Percutaneous Ultrasound-guided Cholecystocentesis and Bile Analysis for the Detection of Platynosomum spp.- Induced Cholangitis in Cats. Journal of Veterinary Internal Medicine, 30(3): 787793, 2016.

Mamprim, M.J; Sartor, R. Fígado e vesícula biliar. In: Carvalho, C.F. Ultrassonografia em pequenos animais. $2^{\mathrm{a}}$ ed. São Paulo: Roca, 2016. p. 61-87.

Michaelsen, R.; Silveira, E.; Marques, S.M.T.; Pimentel, M.C.; Costa, F.V.A. Platynosomum concinnum (Trematoda: Dicrocoeliidae) em gato - doméstico da cidade de Porto Alegre, Rio Grande do Sul, Brasil. Veterinária em Foco, 10(1): 53-60, 2012.

Montserin, S.A.S., Muñoz, K.; Seebaransingh, R.; Basu, A.K. Clinical case: Platynosomum fastosum Kossack, 1910 infection in a cat: First reported case in Trinidad and Tobago. Revue de Médecine Vétérinaire, 164(1): 9-12, 2013.

Norsworthy, G.D. Fascíolas hepáticas, biliares e pancreáticas. In: Norsworthy, G.D.; Crystal, M.A.; Grace, S.F.; Tilley, L.P. O paciente felino: tópicos essenciais de diagnóstico e tratamento. $2^{\mathrm{a}}$ ed. São Paulo: Manole, 2003. p. 304-310.

Pimentel, D.C.G.; Amorim, F.V.; Calixto, R.S.; Faria, V.P. Encefalopatia hepática por platinossomíase: relato de caso. Revista de Educação Continuada da Associação de Clínicos Veterinários de Pequenos Animais, 3(1): 209-211, 2005.
Ramos, R.A.N.; Lima, V.F.S.; Monteiro, M.F.M.; Santana, M.A.; Leopold, R.; Faustino. M.A.G.; Rinaldi, L.; Cringoli, G.; Alves, L.C. New insights into diagnosis of Platynosomum fastosum (Trematoda: Dicrocoeliidae) in cats. Parasitology Research, 115(2): 479-482, 2016.

Ramos, D.G.S.; Santos, A.R.G.L.O.; Freitas, L.C.; Braga, I.A.; Silva, E.P.; Soares, L.M.C.; Antoniassi, N.A.B.; Furlan, F.H.; Pacheco, R.C. Feline platynosomiasis: analysis of the association of infection levels with pathological and biochemical findings. Revista Brasileira de Parasitologia Veterinária, 26(1): 54-59, 2017.

Rocha, N.O.; Portela, R.W.; Camargo, S.S.; Souza, W.G.; Carvalho, G.C.; Bahiense, T.C. Comparison of two coproparasitological techniques for the detection of Platynosomum sp. infection in cats. Veterinary Parasitology, 204(3-4): 392-395, 2014.

Sampaio, M.A.S.; Berlim, C.M.; Angelim, A.J.G.L.; Gondim, L.F.P.; Almeida, M.A.O. Infecção natural pelo Platynosomum Looss 1907, em gato no município de Salvador, Bahia. Revista Brasileira de Saúde e Produção Animal, 7(1): 01-06, 2006.

Salomão, M.; Dantas, L.M.S.; Almeida, F.M.; Branco, A.S.; Bastos, O.P.M.; Sterman, F.; Labarthe, N. Ultrasonography in hepatobiliary evaluation of domestic cats (Felis catus, L. 1758) infected by Platynosomum Loss, 1907. The International Journal of Applied Research in Veterinary Medicine, 3(3): 271279, 2005.

Shell, L.; Ketzis, J.; Hall, R.; Rawlins, G.; Plessis, W. Praziquantel treatment for Platynosomum species infection of a domestic cat on St Kitts, West Indies. Journal of Feline Medicine and Surgery Open Reports, 1(1): 1-4, 2015.

Silva, J.C.S.; Costa, A.P; Praseres, D.C.; Torres, M.A.O.; Oliveira Neta, M.D.; Teófilo, T.S. Endoparasitas em cães e gatos diagnosticados em São Luís-Maranhão. PUBVET, 11(6): 538645, 2017.

Sobral, M.C.G.O.; Sousa, S.A.P.; Ribeiro, T.M.P; Galvão, S.R.; Santos, R.M.; Silva, R.A.; Reis, T.S.; Dias, F.E.F.; Santos, H.D. Infecção por Platynosomum illiciens $(=P$. fastosum $) \mathrm{em}$ gatos domésticos de Araguaína, Tocantins, Norte do Brasil. Revista Brasileira de Parasitologia Veterinária, 28(4): 786-789, 2019. 
Soldan, M.H.; Marques, S.M.T. Platinosomose: Abordagem na clínica felina. Revista da FZVA, 18(1): 46-67, 2011.

Stockham, S.L.; Scott, M.A. Fundamentos de patologia clínica veterinária. Rio de Janeiro: Guanabara Koogan, 2011. 760p.

Sousa Filho, R.P.; Sampaio, K.O.; Holanda, M.S.B.; Vasconcelos, M.C.; Morais, G.B.; Viana, D.A.; Costa, F.V.A. Primeiro relato de infecção natural pelo Platynosomum spp. em gato doméstico no Município de Fortaleza, Ceará, Brasil. Arquivos de Ciências Veterinárias e Zoologia da UNIPAR, 18(1): 59-63, 2015.

Weiser, G.; Thrall, M.A. Considerações sobre leucócitos e leucograma. In: Thrall, M.A.;
Baker, D.C.; Campbell, T.W.; Denicola, D.; Fettman, M.J.; Lassen, E.D.; Rebar, A.; Weiser, G. Hematologia e bioquímica clínica veterinária. $1^{a}$ ed. São Paulo: Roca, 2007. p. 118-122.

Vieira, A.L.S.; Ecco, R.; Lima, W.S.; Guedes, R.M.C. Platynosomum fastosum infection in two cats in Belo Horizonte, Minas Gerais State, Brazil. Brazilian Journal of Veterinary Pathology, 2(1): 45-48, 2009.

Xavier, F.G.; Morato, G.S.; Righi, D.A.; Maiorka, P.C.; Spinosa, H.S. Cystic liver disease related to high Platynosomum fastosum infection in a domestic cat. Journal of Feline Medicine and Surgery, 9(1): 51-55, 2007. 